Veterinary Research Communications, 28 (2004) 385-388

(C) 2004 Kluwer Academic Publishers. Printed in the Netherlands

\title{
Serum Values of Cardiac Troponin-T in Normal and Cardiomyopathic Dogs
}

A. Tarducci*, O. Abate, M. Borgarelli, A. Borrelli, R. Zanatta and A. Cagnasso Department of Animal Pathology, Faculty of Veterinary Medicine, University of Torino, Grugliasco (To), Italy

* Correspondence: Dipartimento di Patologia Animale - Facoltà di Medicina

Veterinaria, Via L. da Vinci, 44 - 10095 Grugliasco ( Torino) - Italy

E-mail: alberto.tarducci@unito.it

Keywords: troponin T, dog, heart, dilated cardiomyopathy, ventricular hypokinesis

\section{INTRODUCTION}

Together with tropomyosin, the troponin complex participates in the contraction of skeletal and cardiac muscles. Troponin is composed of three subunits, namely troponin $\mathrm{C}(\mathrm{TnC})$, troponin $\mathrm{I}(\mathrm{TnI})$, and troponin $\mathrm{T}(\mathrm{TnT})$, which binds tropomyosin. There are three different isoforms of TnT and TnI, two of which are specific for skeletal muscle, while the third is specific for the myocardium (cTnT and cTnI). As the homology between troponins is about $95 \%$ among mammals, commercial diagnostic kits designed for use in humans also provide excellent results in other animals. (O'Brien et al., 1997).

In human medicine, the measurement of $\mathrm{cTnT}$ or $\mathrm{cTnI}$ levels is now used together with measurement of the levels of creatine kinase (CK) and lactate dehydrogenase $(\mathrm{LDH})$ and their isozymes for assessment of myocardial damage. For diagnostic purposes, one advantage is that these proteins are normally absent in blood in subjects without myocardial damage. Even minimal increases in the serum levels of these proteins are indicative of myocardial damage and, in the case of $\mathrm{cTnT}$, it is detectable in serum from $2 \mathrm{~h}$ to 14 days following the onset of myocardial damage. Moreover, in contrast to the isozymes of $\mathrm{CK}$ and $\mathrm{LDH}$, the results are not influenced by use of different clinical samples (hemolytic, lipemic, bile samples). The degree of increase in the level of cTnT is relatively proportional to the degree of myocardial damage, with prognostic implications as well. At the same time, the extreme sensitivity of this technique also allows for its use in other nonischemic pathologies including chronic cardiac insufficiency, pulmonary emboli, acute myocarditis, systemic hypertension, arrhythmias, and toxicity from doxorubicin (De Francesco, 2002). In veterinary medicine, O'Brien et al. (1997) have demonstrated the utility of measuring the levels of cTnT in the diagnosis of experimentally induced myocardial damage.

\section{MATERIALS AND METHODS}

cTnT levels were determined using an immunochemical test developed for humans using whole, heparinized venal blood (Cardiac Reader, Roche) within $2 \mathrm{~h}$ of blood 
collection. Values of cTnT less than 0.05 were considered negative (expressed in $\mathrm{ng} / \mathrm{ml}$ ). Values of cTnT between $0.05-0.1,0.1-2$, and $>2$ were considered as low, intermediate, and high, respectively. The cTnT levels were measured for a control group of $20 \mathrm{dogs}$ that were judged as healthy on the basis of history and objective clinical examination with particular attention to the cardiovascular system. This group of animals comprised subjects with different races and gender with an age between 1 to 12 years (mean 6.1). Another group of 30 animals was defined as cardiomyopathic on the basis of clinical and instrumental examination (e.g., echocardiograph and ecoDoppler, radiography and EKG). These all had cardiac insufficiencies between $1 \mathrm{~b}$ and $3 \mathrm{a}$, according to the ISACHC classification. On the basis of echocardiographic examination, the myocardiopathic group was further divided into two subgroups: non-hypokinetic myocardiopathic ( 12 dogs aged between 3 months and 16 years, mean 7.5) and hypokinetic myocardiopathic (global or segmental hypokinetic; 18 dogs aged between 7 months and 15 years, mean 6.0). Statistical analysis using Fisher's test was employed to evaluate the correlation between a positive cTnT test and cardiac insufficiency, as well as ventricular kinetics. The aim of the present work was to determine the value of cTnT for healthy dogs and for dogs affected with different cardiac pathologies, evaluating eventual correlations between cTnT levels, the ISACHC classification for cardiac insufficiency, the index of systolic volume (ESV-I), and ventricular kinetics.

\section{RESULTS}

The control group of healthy dogs had values of serum cTnT that were below detectable levels (i.e., $<0.05 \mathrm{ng} / \mathrm{ml}$ ). Ten of the 30 myocardiopathic dogs $(33 \%)$ tested positive for cTnT. However, there was no significant correlation between a positive result in the cTnT assay and the type of cardiac insufficiency or with the ESV-I. In the non-hypokinetic group, only one of the $12 \mathrm{dogs}(8.3 \%)$ which had an intermediate level of cTnT was positive. In the hypokinetic myocardiopathic group, 9 out of 18 animals tested positive, three with low levels of cTnT, four with intermediate levels, and two with high levels. The probability of a positive cTnT value was significantly higher for hypokinetic animals with respect to non-hypokinetic dogs (Fisher's exact test, $\mathrm{OR}=10, p<0.05$ ).

It was possible to carry out a second examination for 4 out of 10 dogs at follow-up visits during the course of therapy. In three animals, the cTnT level was below detectable levels by the second control visit and was associated with an improvement in general clinical conditions in all cases. The remaining dog, diagnosed with dilative myocardiopathy, had levels of cTnT that had increased over a two week interval, although they still fell in the intermediate value category. During this period, the dog's conditions progressively worsened in spite of therapy and the animal died three days after the last visit. For the three dogs with dilative myocardiopathy, the value of cTnT was below detection limits. 


\section{DISCUSSION}

The absence of detectable cTnT $(>0.05 \mathrm{ng} / \mathrm{ml})$ in healthy dogs confirms the utility of this assay in assessing acute myocardial damage, similar to that reported both for both human and veterinary subjects (De Francesco, 2002). The results of the present work indicate the usefulness of the cTnT test for the identification of cardiopathic subjects for whom disease is associated with myocardial damage. Furthermore, our data are also interesting in relation to the lack of correlation between a positive cTnT result and the degree of systolic insufficiency. In addition, the observation that only $50 \%$ of the hypokinetic group was positive for cTnT suggests that the assay may have particular utility in discriminating animals with myocardial damage in course from those in which hypokinesis is most likely indicative of a previous injury. On the other hand, the positive test results observed in non-hypokinetic, myocardiopathic subjects implies that the measurement of cTnT is a diagnostic tool that is valuable for the identification of myocardial damage, even in animals that would not otherwise be suspected to have myocardial damage on the basis of clinical and instrumental examination.

The determination of cTNT during the follow-up period may also have prognostic implications. During this period, clinical improvement was seen in all three 3 cases with negative cTnT, while in the subject for whom increased levels of cTnT were observed, clinical deterioration was noted and resulted in sudden death. Lastly, for the three dogs with dilative myocardiopathy serum cTnT was not detected. This may be explained by the low concentrations of troponin $\mathrm{T}$ at the myocardial level, as reported by O'Brien (1997) for Dobermans affected with idiopathic dilative myocardiopathy. Our results are in contrast to those found for humans, in which a relatively high percentage of patients were found to be positive for cTnT during the course of dilative myocardiopathy. It has been reported that the return to normal levels of cTnT following therapy also has a beneficial impact on prognosis, with survival times that are significantly higher (Sato et al., 2001). However, in the present study the number of animals with dilative myocardiopathy, all with negative cTnT, is too small to make any conclusions in this regard.

\section{ACKNOWLEDGEMENTS}

This work was supported by grants from Italian MURST.

\section{REFERENCES}

De Francesco, T.C., Atkins, C.E., Keene, B.W., Coats, J.R. and Hauck, M.L., 2002. Prospective clinical evaluation of serum cardiac troponin $\mathrm{T}$ in dogs admitted to a veterinary teaching hospital. Journal of Veterinary Internal Medicine, 16, 553-557

O'Brien, P.J., 1997. Deficiencies of myocardial troponin-T and creatine kinase MB isoenzyme in dogs with idiopathic dilated cardiomyopathy. American Journal Veterinary Research, 58, 11-16 
O’Brien P.J. Dameron, G.W., Beck, M.L., Kang, Y.J., Erickson, B.K., Di Battista, T.H., Miller, K.E., Jackson, K.N. and Mittelstadt, S., 1997. Cardiac troponin T is a sensitive, specific biomarker of cardiac injury in laboratory animals. Laboratory Animal Science, 47, 486-495

Sato, Y., Yamada, T., Taniguchi, R., Nagai, K., Makiyama, T., Okada, H., Kataoka, K., Ito, H. Matsumori, A., Sasayama, S. and Takatsu, Y., 2001. Persistently increased serum concentrations of cardiac Troponin $\mathrm{T}$ in patients with idiopathic dilated cardiomyopathy are predictive of adverse outcomes. Circulation, 103, 369-374 